\section{Zur Typologisierung der Abhängigkeit von legalen Suchtmitteln bei Frauen}

Christel Zenker

Fachhochschule Münster, FB Sozialwesen
Zusammenfassung: Zur Optimierung therapeutischen und präventiven Handelns ist die Differenzierung weiblicher Abhängigkeit notwendig. Die Aufnahmegespräche mit 397 stationär zur Entwöhnung aufgenommenen Frauen wurden hinsichtlich suchtauslösender und suchtbegleitender Faktoren quantitativ ausgewertet. Es konnten fünf Gruppen auf der Grundlage belastender und die persönliche Entwicklung einschränkender Lebensereignisse identifiziert werden: Drei Gruppen unterscheiden sich durch die Intensität sozialer Belastungen und Gewalterfahrungen, die wiederum mit dem Suchtbeginn, den gewählten Suchtformen und -mitteln sowie seelischen Störungen korrelieren. Bei der vierten Gruppe scheint ein einschneidendes Lebensereignis suchtbestimmend, in der fünften Gruppe bilden Essstörungen auf der Grundlage seelischen Missbrauchs in der Kindheit das Hauptsymptom.

Schlüsselwörter: Sucht - Frauen - Ursachen - Typologisierung

Typology of Drug Dependency in Women: To improve the knowledge about drug dependency in women the first interviews with 397 women, admitted to a hospital for women dependent on legal drugs, were quantitatively evaluated. Five groups could be formed. Three groups differ in onset and extent of dependent behaviour, which is related to the experience of sexual, psychic and physical abuse, formerly treated psychic disorders and drug misuse in the social environment of the women. Most women aged between 15-20 years when first using drugs or developing eating problems did suffer all forms of abuse, showed a polytoxic behaviour and had been treated because of psychic disorders whereas women, starting drug use when between 35-50 years old, were dependent only on alcohol, did only experience mistreatment in their adult life and had not experienced psychic disorders. In a fourth group a traumatic life event caused the dependency, for which, on the basis of negative psychic experiences in childhood, no other coping strategies could be developed. In a fifth group of very young women, with the experience of emotional-psychic abuse in childhood, eating disorders were the leading symptoms of a polytoxic behaviour and were accompanied by a relatively high proportion of psychic disorders.

Suchttherapie 2001; 2: 28-34

(c) Georg Thieme Verlag Stuttgart · New York ISSN 1439-9903
Key words: Addiction - Women - Typology - Causes

\section{Einleitung}

Alkoholkranke stellen keine homogene Population dar, dies belegen zahlreiche Klassifikations- und Typologieversuche. Preuß et al. haben 1997 in einem kritischen Vergleich darauf hingewiesen, dass es eine komplette Erfassung von Alkoholkranken in Untergruppen nicht geben kann, Typologien jedoch sinnvoll seien, da sie Unterschiede zwischen Subgruppen für Forschung und Therapie aufdecken [1].

Während der Verlauf der Alkoholkrankheit eher geschlechtsunabhängig zu sein scheint [2], wurden geschlechtsspezifische Ursachen des Alkoholmissbrauchs näher beleuchtet: Seit Beginn der 90er Jahre wird in europäischen und außereuropäischen Untersuchungen auf spezifische lebensweltliche Erfahrungen wie körperlichen und sexuellen Missbrauch bei der Entstehung der Sucht bei Frauen hingewiesen, s.a. [3-8]. In einem Review des Amsterdam Institute for Addiction Research wird der Einfluss von körperlichem und sexuellem Missbrauch in der Kindheit bei Frauen, aber nicht bei Männern als ursächlich für die Entwicklung späterer Alkoholprobleme hervorgehoben [9]. Vogt hat 1986 zwei Alkoholikerinnentypen unterschieden: Diejenigen, die um das zweite Lebensjahrzehnt, und diejenigen, die um das dritte Lebensjahrzehnt mit dem schädigenden Alkoholkonsum beginnen und sich durch die Schwere ihres Krankheitsbildes unterscheiden [10].

\section{Untersuchungsziel und Methodik}

Die therapeutisch tätigen Mitarbeiterinnen und Mitarbeiter in Suchthilfeeinrichtungen stützen sich bei antizipierten Behandlungsverläufen und Prognosekriterien auf über Jahre gewachsene Erfahrungswerte. Bei den Frauen stellen Gewalterfahrungen, das Alter bei Suchtbeginn und gewählte Suchtmittel wesentliche Bewertungskriterien dar. Ziel der Untersuchung war es, diese Erfahrungen durch Quantifizierung bestimmter Merkmale bei den behandelten Frauen zu ordnen und unterschiedliche Gruppen zu identifizieren.

Retrospektiv wurden die ca. einstündigen Erstexplorationsgespräche ausgewertet, die die Autorin als leitende Ärztin mit 397 Frauen geführt hat, die erstmalig in ihrem Leben und routinemäßig zwischen 1995-1997 in einer Fachklinik für abhängigkeitskranke Frauen aufgenommen wurden. Die Form 
der Erstexploration entspricht am ehesten einem strukturierten Interview. Der Vorteil der Anamneseerhebung gegenüber einer anonymen Fragebogen-Aktion liegt in der Möglichkeit zur Beziehungsaufnahme und damit Nachfrage und Klärung des Gesagten: Die Zuordnung als neutrale, positive oder negative Einflussfaktoren auf die persönliche Entwicklung und Gestaltung ihres Lebens erfolgte somit durch die Frauen selbst.

\section{Variablen zur Gruppenbildung}

Grundlage der Bildung unterschiedlicher Gruppen sind die Variablen: soziale Einflussfaktoren und Gewalterfahrungen seit der Kindheit, Gewalt ausübende Personen, süchtige Personen im früheren und späteren sozialen Umfeld und einschneidende Lebensereignisse. Als „negative soziale Einflussfaktoren“ wurden anhaltende Lebensumstände gewertet, die materielle und soziale Stabilität und Stabilität in den persönlichen Beziehungen vermissen ließen (z.B. materielle Not, wechselnde Bezugspersonen, Übernahme von Verantwortung für jüngere Geschwister), als entlastende oder Schutzfaktoren wurden solche gewertet, die die Identitätsbildung und Ich-Stärkung der Frauen unterstützten (z.B. Zusammenhalt von Geschwistern, liebevoller Elternteil). „Gewalterfahrungen“ wurden nach Häufigkeit und Art in körperliche (Schläge, Prügel mit Verletzungsfolgen, Quälereien) und seelische (Herabsetzung, Kränkung, Unterwerfung, emotionale Ausbeutung und Abweisung, seelische Kälte und die Erfahrung, unerwünscht zu sein) unterteilt. „Sexueller Missbrauch" in der Kindheit beinhaltet erzwungene sexuelle Berührungen bis zum vollzogenen Geschlechtsverkehr, die das Geschehen begleitenden Gefühle wie Angst, Scham, Schuld, Ekel oder Verwirrung, „Sich-Wegmachen“, „Sich-Abspalten“, Selbstverletzungen sowie die Dauer dieser Erfahrungen. Erlittene sexuelle Gewaltanwendungen im Erwachsenenalter wurden als „Vergewaltigungen“ kategorisiert. Als „Sucht im sozialen Umfeld“ wurde ein die Mädchen und/oder Frauen beeinträchtigender Suchtmittel-, meist Alkoholmissbrauch gewertet, besonders häufig im „männlichen“ sozialen Umfeld (z.B. den betrunkenen Vater regelmäßig aus Kneipen holen). „Einschneidende Lebensereignisse“ sind der Tod eines (meist einzigen) geliebten, emotional stützenden Angehörigen oder Tod, Behinderung bzw. chronische Erkrankung eines eigenen Kindes.

\section{Gruppenbildung}

Art und Häufigkeit negativ und positiv bewerteter Variablen sind Grundlage für die Bildung von drei Gruppen, die Schweregrade der „persönlichen Beschädigungen“ der Frauen abbilden. Ein außergewöhnliches und in seiner Folge emotional belastendes und nicht integrierbares Lebensereignis ist Hauptmerkmal der vierten und emotionale Missbrauchserfahrungen in der Kindheit wichtigstes Merkmal der fünften Gruppe.

Neben diesen Sucht auslösenden Faktoren können weitere Merkmale als typisch für die einzelnen Gruppen beschrieben werden: Suchtform und Suchtmittel, Alter bei Suchtbeginn, Schul- und Berufsbildung, sexuelle Aktivitäten, die Neigung, selbst Gewalt auszuüben, sowie ambulant oder stationär vor der Klinikaufnahme behandelte oder das Leben der Frauen über Jahre nachhaltig beeinträchtigende psychische Störungen in der Vorgeschichte.

Die einzelnen, gruppentypischen Faktoren und Merkmale bedingen sich meist gegenseitig; so ist die Gewaltbereitschaft des Vaters u.a. oft mit dessen Alkoholmissbrauch, existenzieller Not der Familie und unzureichender Ausbildung der Tochter verbunden oder die als intensiv und zerstörerisch erlebte Gewalterfahrung ist u.a. mit einem polytoxikomanen und früh im Leben beginnenden Suchtmittelkonsum und schwereren seelischen Störungen vergesellschaftet. Bei fraglicher Gruppenzuordnung wurde der Gesamteindruck bei der Exploration mit berücksichtigt. Insgesamt erscheint Gewalterfahrung als ein charakteristisches Merkmal für die einzelnen Gruppen, die in Tab. 1 nach ihrem Anteil an Gewalterfahrung vorgestellt werden.

\section{Gruppenmerkmale}

Die folgenden Gruppenbeschreibungen heben die jeweils typischen Merkmale hervor.

Gruppe I (psychosoziale Schädigung in der Kindheit, Folge: selbstzerstörerische Tendenzen) $(\mathrm{n}=140)$ ist durch massive soziale, psychische, physische, und sexuelle Beschädigungen ohne emotionalen Ausgleich in der Kindheit sowie frühe Suchtentwicklung mit polytoxikomanen Tendenzen gekennzeichnet. Wechselnde Bezugspersonen, Pflege- und Heimaufenthalte oder sehr abgeschottete zerstörerische Familiensysteme sind typisch. Im späteren Leben fallen frühe sexuelle Erfahrungen, instabile gewaltvolle Partnerbeziehungen, massive soziale Probleme und oft Gewalt gegenüber den eigenen Kindern sowie deren Fremdunterbringung auf. In der psychiatrischen Anamnese werden mehrfache Suizidversuche, Behandlungen wegen Depressionen, Ängsten, psychosomatischen Erkrankungen, Persönlichkeitsstörungen und Psychosen genannt. Die Frauen empfinden sich als affektiv tot und neigen zu Selbstverletzungen.

\begin{tabular}{|c|c|c|c|c|c|c|}
\hline \multirow[t]{2}{*}{ Gruppen } & \multicolumn{2}{|l|}{ Frauen } & \multicolumn{2}{|c|}{ mit Gewalterfahrung } & \multicolumn{2}{|c|}{ ohne Gewalterfahrung } \\
\hline & $\mathrm{n}$ & $\%$ & $\mathrm{n}$ & $\%$ & $\mathrm{n}$ & $\%$ \\
\hline Gruppe I & 140 & 35,3 & 133 & 95,0 & 7 & 5,0 \\
\hline Gruppe II & 161 & 40,6 & 127 & 78,9 & 34 & 21,1 \\
\hline Gruppe III & 43 & 3,0 & 20 & 46,5 & 23 & 53,5 \\
\hline Gruppe IV & 12 & 10,8 & 4 & 33,3 & 8 & 66,7 \\
\hline Gruppe V & 41 & 10,3 & 26 & 63,4 & 15 & 36,6 \\
\hline alle & 397 & 100 & 310 & 78,1 & 87 & 21,9 \\
\hline
\end{tabular}

Tab. 1 Gruppen nach Gewalterfahrung 
Gruppe II (psychosozial gefährdetes Leben, Folge: Tendenz zur Selbstaufgabe) ( $\mathrm{n}=161)$ ist vor allem durch körperliche, aber auch seelische Gewalterfahrungen, Entbehrungen und Unterdrückung in einem sozial belasteten Umfeld, mit frühzeitiger Übernahme von Verantwortung für andere Familienmitglieder, aber auch durch emotionalen Ausgleich in der Kindheit gekennzeichnet. Suchtbeginn im frühen Erwachsenenleben, frühe Eheschließung, gewaltvolle Partnerbeziehungen, Eheund Erziehungsschwierigkeiten sowie berufliche Probleme bestimmen das spätere Leben. Als objektiv und subjektiv zu kurz Gekommene nehmen sie eine Opferrolle ein; Selbstverleugnung, Suizidversuche, Depressionen und Ängste sind typisch.

Gruppe III (unterdrücktes, abhängiges Leben, Folge: mangelndes Selbstwertgefühl) $(\mathrm{n}=43)$ ist durch Anpassungsdruck in Kindheit und Erwachsenenleben mit verinnerlichter Unselbständigkeit gekennzeichnet. Kränkungen, Verachtung und physische Gewalt durch den Partner sind ebenso typisch wie die Leugnung eigener Bedürfnisse und (vor allem als negativ erachteter) Gefühle; Suchtbeginn im späteren Erwachsenenleben. Seelische Stabilität wird aus angepasstem Verhalten gezogen, psychosomatische Störungen und Depressionen sind häufig.

Gruppe IV (life event, Folge: Scheitern an außergewöhnlicher Belastung) ( $\mathrm{n}=12$ ) ist durch eine instabile (emotional, sozial) Kindheit, oft mit Sucht im sozialen Umfeld gekennzeichnet. Auf der Grundlage mangelnder Selbstbehauptung und Bestätigung führt ein einschneidendes Lebensereignis in der Kindheit (z.B. vermeintliche Schuld am Tod eines Geschwisters) oder im Erwachsenenleben (z.B. chronische Erkrankung, Behinderung eines Kindes), für das keine ausreichenden Copingstrategien zur Verfügung stehen, zur Sucht im frühen oder mittleren Erwachsenenleben. Seelische, vor allem depressive Störungen sind häufig vorhanden.

Gruppe V (psychische Überforderung im Reifungsprozess, Folge: Flucht $)(\mathrm{n}=41)$ ist durch seelisch-emotionale Gewalt in Kindheit und Jugend, durch ablehnende oder stark bindende oder keine Orientierung gebende Eltern, oft in einem sozial stabilen Umfeld gekennzeichnet. Essstörungen (Magersucht/ Esssucht) und/oder polytoxikomanes Verhalten werden als Protest und Hilferuf eingesetzt. Unterbrochene schulische oder berufliche Laufbahn bei emotional und sozial instabilem Verhalten mit Suizidversuchen, Depressionen und Selbstverletzungen sind typisch.

In Tab. 2 wurden die typischen Merkmale der Gruppen in der Übersicht dargestellt. Zahlen und Prozentwerte wurden gerundet.

\section{Auswertungsergebnisse}

\section{Alter bei der Aufnahme}

Zum Aufnahmezeitpunkt waren die 397 Frauen zwischen 17 und 69 Jahre alt, die 30- bis 49-Jährigen stellten mit 64,8\% (30-39 J. 33,8\%; 40-49 J. 31,0\%) die Hauptgruppe der Behandelten, die 20 - bis 29-Jährigen 18,6\% der Gesamtgruppe. Jünger als 20 Jahre waren 9 (2,3\%), älter als 60 Jahre $10(2,5 \%)$ Frauen.

\section{Erste Entwöhnungsbehandlung}

Frauen, die in jungen Jahren Essstörungen entwickelten (10-19 Jahre, Gruppe V) oder spät in ihrem Leben abhängig wurden (35-50 Jahre, Gruppe III), kommen relativ schnell, 5-10 Jahre nach Suchtbeginn erstmals in Entwöhnungsbehandlung. Frauen mit lebenslanger Gewalterfahrung und Suchtentwicklung zwischen dem 10. und 39. Lebensjahr kommen 10-15 Jahre nach Suchtbeginn in Entwöhnungsbehandlung. Der Zeitraum zwischen Suchtbeginn und Behandlung ist in Gruppe IV (life event) uneinheitlich.

\section{Suchtbeginn}

Das Alter beim Einstieg in süchtiges Verhalten kann als Maß für die erlittenen Kränkungen und seelischen Beschädigungen der Frauen angesehen werden. Gewalterfahrungen sind ein wichtiger, aber nicht ausschließlicher Indikator für diese Kränkungen. Von 397 Frauen gaben 310 (78,1\%) an, auch

Tab. 2 Merkmale der Gruppen in der Übersicht

\begin{tabular}{|c|c|c|c|c|c|c|c|}
\hline $\begin{array}{l}\text { Gruppe } \\
\text { (Gruppen- } \\
\text { größe) }\end{array}$ & $\begin{array}{l}\text { typisches } \\
\text { Alter bei } \\
\text { Suchtbeginn }\end{array}$ & $\begin{array}{l}\text { Anteil mit } \\
\text { Gewalt- } \\
\text { erfahrungen }\end{array}$ & $\begin{array}{l}\text { Gewalt vorwie- } \\
\text { gend in Lebens- } \\
\text { abschnitt }\end{array}$ & $\begin{array}{l}\text { typische } \\
\text { Gewaltform }\end{array}$ & häufigste Suchtmittel & $\begin{array}{l}\text { Sucht im } \\
\text { persönlichen } \\
\text { Umfeld }\end{array}$ & $\begin{array}{l}\text { Vorkommen } \\
\text { psychischer } \\
\text { Störungen }\end{array}$ \\
\hline $\begin{array}{l}\text { Gruppe I } \\
(n=140)\end{array}$ & $15-20 \mathrm{~J}$. & $95 \%$ & lebenslang & $\begin{array}{l}\text { sex. Missbrauch, } \\
\text { seelisch-körperliche, } \\
\text { Vergewaltigung }\end{array}$ & $\begin{array}{l}\text { Alkohol, Alk. komb. } \\
\text { mit Med., Essstörungen, } \\
\text { illegalen Drogen }\end{array}$ & $60 \%$ & $70 \%$ \\
\hline $\begin{array}{l}\text { Gruppe II } \\
(\mathrm{n}=161)\end{array}$ & $30-35 \mathrm{~J}$. & $80 \%$ & lebenslang & $\begin{array}{l}\text { seelisch- } \\
\text { körperliche }\end{array}$ & $\begin{array}{l}\text { Alkohol, Alk. komb. } \\
\text { mit Medikamenten, } \\
\text { Essstörungen }\end{array}$ & $50 \%$ & $30 \%$ \\
\hline $\begin{array}{l}\text { Gruppe III } \\
(\mathrm{n}=43)\end{array}$ & $35-50 \mathrm{~J}$ & $45 \%$ & $\begin{array}{l}\text { Erwachsenen- } \\
\text { leben }\end{array}$ & körperlich-seelische & Alkohol & $25 \%$ & $10 \%$ \\
\hline $\begin{array}{l}\text { Gruppe IV } \\
(\mathrm{n}=12)\end{array}$ & $\begin{array}{l}25-35 \mathrm{~J} . \\
\text { (oder später) }\end{array}$ & $35 \%$ & Kindheit & seelisch-körperliche & Alkohol & $40 \%$ & $40 \%$ \\
\hline $\begin{array}{l}\text { Gruppe V } \\
(n=41)\end{array}$ & $10-15 \mathrm{~J}$. & $65 \%$ & Kindheit & $\begin{array}{l}\text { seelisch-emotionale } \\
\text { (sex. Missbrauch) }\end{array}$ & $\begin{array}{l}\text { Essstörungen, Essst. } \\
\text { komb. mit Alk., illeg. } \\
\text { Drogen, Medikamenten }\end{array}$ & $20 \%$ & $40 \%$ \\
\hline
\end{tabular}




\begin{tabular}{|c|c|c|c|c|c|c|}
\hline \multirow{2}{*}{$\begin{array}{l}\text { Alter bei } \\
\text { Suchtbeginn } \\
\text { Jahre }\end{array}$} & \multicolumn{2}{|l|}{ Frauen } & \multicolumn{2}{|c|}{$\begin{array}{l}\text { davon: } \\
\text { Gewalterfahrung }\end{array}$} & \multicolumn{2}{|c|}{$\begin{array}{l}\text { davon: keine Gewalt- } \\
\text { erfahrung }\end{array}$} \\
\hline & $n$ & $\%$ & $\mathrm{n}$ & $\%$ & $\mathrm{n}$ & $\%$ \\
\hline$<10$ & 7 & 1,7 & 7 & 100 & & \\
\hline $10-14$ & 38 & 9,6 & 33 & 86,8 & 5 & 13,2 \\
\hline $15-19$ & 77 & 19,4 & 65 & 84,4 & 12 & 15,6 \\
\hline $20-29$ & 112 & 28,2 & 90 & 80,4 & 22 & 19,6 \\
\hline $30-39$ & 107 & 27,0 & 82 & 76,6 & 25 & 23,4 \\
\hline $40-49$ & 41 & 10,3 & 28 & 68,3 & 13 & 31,7 \\
\hline $50-59$ & 15 & 3,8 & 5 & 33,3 & 10 & 66,7 \\
\hline alle & 397 & 100 & 310 & 78,1 & 87 & 21,9 \\
\hline
\end{tabular}

Tab. 3 Alter bei Suchtbeginn und Gewalterfahrung
Gewalt erfahren zu haben. Jede Frau, die schon vor dem 10. Lebensjahr Suchtmittel konsumiert hatte, gab an, vorher Gewalt erfahren zu haben. Demgegenüber haben 66,7\% der Frauen, die nach dem 50. Lj. mit dem Konsum begonnen haben, keine Gewalterfahrung. Bei ihnen haben andere Faktoren, Demütigungen und Unterdrückung, zur Sucht geführt.

Diejenigen, die als Kinder/Jugendliche mit dem Suchtmittelkonsum begannen, haben Alkohol und/oder Medikamente oft von den Gewalttätern, zur Erleichterung des sexuellen Missbrauchs, wie in Gruppe I, erhalten oder sie haben, wie in Gruppe V, Essstörungen als Ausdruck des Protestes gegen (Anorexie) oder des Sich-Fügens in (Esssucht) emotionale Gewalt entwickelt (Tab. 3).

\section{Gewalterfahrungen}

Unterschieden wurde zwischen sexuellem Missbrauch, der zumindest seelische, oft körperliche Gewaltausübung einschließt, und seelisch-körperlicher Gewalt ohne sexuellen Missbrauch. Körperliche Gewalt wurde immer auch als seelische Kränkung erfahren bzw. war mit ihr kombiniert, während seelische Gewalt ohne körperliche Grenzverletzung auskommt.

Je frühzeitiger, intensiver und regelmäßiger Gewalt erfahren wurde, desto häufiger wurde hierauf mit seelischen Störungen schon in der Kindheit (Entwicklungs-, Lern-, Verhaltens-, psychosomatische Störungen) reagiert und/oder es wurden süchtige Poblemlösungsstrategien entwickelt. Seelische Gewalt wurde von den Frauen schwer wiegender und einschneidender erlebt als körperliche Gewalt, sexueller Missbrauch umso zerstörerischer, je früher im Leben der Frauen er begann und je unausweichlicher er war, d.h., je intensiver er das Leben des Kindes bestimmte und je seltener ein emotionaler Ausgleich durch eine andere Bezugsperson gegeben war.

\section{Gewalterfahrungen nach Lebensabschnitten}

Von den 310 Frauen mit Gewalterfahrung haben 164 Frauen $(52,9 \%)$ Gewalt nur in der Kindheit, 82 Frauen (26,5\%) Gewalt in Kindheit und Erwachsenenleben und 64 Frauen (20,6\%) Gewalt nur im Erwachsenenalter erfahren.

Den höchsten Anteil an Frauen, die Gewalterfahrungen während ihres gesamten Lebens erlitten haben, weisen die Gruppen I und II mit 33,8\% resp. 22,8\% auf. Gruppe III hat den größten Anteil an Frauen, die mit Gewalt nur im Erwachsenenalter konfrontiert waren, 65\% derjenigen mit Gewalterfahrung. Bei Gruppe IV muss die kleine Fallzahl berücksichtigt werden. Gruppe V umfasst Frauen, die sehr jung in die Entwöhnungsbehandlung kommen und in der deshalb der Anteil mit Gewalterfahrung im Erwachsenenalter sehr klein ist. (Tab. 4).

\section{Gewalt in der Kindheit}

In Tab. 5 werden die Gewalt ausübenden Personen nach Nennungen aufgeführt. Die Gruppen III (späte Alkoholabhängigkeit) und IV (life event) wurden nicht berücksichtigt, da deren Anteil an Frauen mit Gewalterfahrung in der Kindheit sehr gering ist.

Bei den „Verwandten“ handelt es sich um im gleichen Haushalt lebende Großväter, seltener Onkel oder Brüder, „Sonstige“ sind vor allem Nachbarn oder Schulkameraden. Die 77 Frauen der Gruppe I benennen 160 Personen oder

\begin{tabular}{|c|c|c|c|c|c|c|}
\hline \multicolumn{3}{|c|}{ Gruppen mit Gewalterf. nur in der Kindheit } & \multicolumn{2}{|c|}{$\begin{array}{l}\text { Kindheit und } \\
\text { Erwachsenenleben }\end{array}$} & \multicolumn{2}{|c|}{$\begin{array}{l}\text { nur im } \\
\text { Erwachsenenleben }\end{array}$} \\
\hline (Anzahl) & $\mathrm{n}$ & $\%$ & $\mathrm{n}$ & $\%$ & $\mathrm{n}$ & $\%$ \\
\hline Gruppe I (n = 133) & 77 & 57,9 & 45 & 33,8 & 11 & 8,3 \\
\hline Gruppe II ( $n=127)$ & 60 & 47,2 & 29 & 22,8 & 38 & 30,0 \\
\hline Gruppe III (n = 20) & 5 & 25,0 & 2 & 10,0 & 13 & 65,0 \\
\hline Gruppe IV (n = 4) & 2 & 50,0 & 1 & 25,0 & 1 & 25,0 \\
\hline Gruppe V $(n=26)$ & 20 & 76,9 & 5 & 19,2 & 1 & 3,8 \\
\hline alle $=310$ & 164 & 52,9 & 82 & 26,5 & 64 & 20,6 \\
\hline
\end{tabular}

Tab. 4 Gewalterfahrung nach Lebensabschnitten 
Tab. 5 Gewalt ausübende Personen in der Kindheit

\begin{tabular}{|c|c|c|c|c|c|c|c|c|c|c|}
\hline \multirow{2}{*}{$\begin{array}{l}\text { Gruppen } \\
\text { (Anzahl) }\end{array}$} & \multicolumn{5}{|c|}{ sexueller Missbrauch durch } & \multicolumn{5}{|c|}{ seelische und/oder körperliche Gewalt durch } \\
\hline & $\begin{array}{l}\text { Vater } \\
\mathrm{n}\end{array}$ & $\begin{array}{l}\text { Mutter } \\
\mathrm{n}\end{array}$ & $\begin{array}{l}\text { Eltern } \\
\mathrm{n}\end{array}$ & $\begin{array}{l}\text { Verwandte } \\
\mathrm{n}\end{array}$ & $\begin{array}{l}\text { Sonstige } \\
\mathrm{n}\end{array}$ & $\begin{array}{l}\text { Vater } \\
\mathrm{n}\end{array}$ & $\begin{array}{l}\text { Mutter } \\
\mathrm{n}\end{array}$ & $\begin{array}{l}\text { Eltern } \\
\mathrm{n}\end{array}$ & $\begin{array}{l}\text { Verwandte } \\
\mathrm{n}\end{array}$ & $\begin{array}{l}\text { Sonstige } \\
\mathrm{n}\end{array}$ \\
\hline Gruppe I (n = 77) & 41 & 11 & 13 & 22 & 14 & 24 & 10 & 18 & 4 & 3 \\
\hline Gruppe II (n = 60) & 6 & 6 & 3 & 7 & 9 & 21 & 23 & 12 & 6 & 6 \\
\hline Gruppe V $(n=20)$ & & & & 1 & 3 & 2 & 5 & 9 & 1 & \\
\hline $\begin{array}{l}\text { Gesamtanzahl } \\
\text { Nennungen }\end{array}$ & 47 & 17 & 16 & 30 & 26 & 47 & 38 & 39 & 11 & 9 \\
\hline
\end{tabular}

beide Eltern, die Gewalt ausgeübt haben. 13 Frauen berichten über sexuellen Missbrauch durch beide Eltern, 11 über sexuellen Missbrauch durch die Mutter allein. Das Verhältnis der Nennungen von sexuellem Missbrauch zu seelisch-körperlicher Gewalt beträgt hier 1,7:1.

In den Gruppen II und V überwiegt die seelisch-körperliche Gewalterfahrung gegenüber dem sexuellen Missbrauch. Im Gegensatz zu Gruppe I wird die Mutter als seelische und/oder körperliche Gewalt ausübende Person häufiger als der Vater genannt (Gruppe II und V) und gleich häufig beim sexuellen Missbrauch (Gruppe II). Bei den Essgestörten (Gruppe V) sind die leiblichen Eltern am (seltenen) sexuellen Missbrauch nicht beteiligt; am häufigsten werden beide Eltern als emotional Gewalttätige genannt.

\section{Suchtmittel}

Alkohol wurde von 350 (88,2\%), Medikamente von 90 (22,6\%), Essstörungen von $84(21,2 \%)$ und illegale Drogen von $52(13,1 \%)$ Frauen als alleinige oder kombinierte Suchtmittel bzw. -formen eingesetzt. Von 3 Frauen wurden ausschließlich illegale Drogen zum Aufnahmezeitpunkt konsumiert, bei früher polytoxikomanem Verhalten. Medikamente spielen als einzige Suchtstoffe (Tranquilizer und Schmerztabletten) insgesamt eine untergeordnete Rolle $(<10 \%)$.

Alkohol ist bei der überwiegenden Anzahl der Frauen (zwischen $75 \%$ bis $86,0 \%$ ) der Gruppen II, III und IV einziger Suchtstoff, während dies in Gruppe I nur bei $40,7 \%$ der Fall ist. Anorexie und Esssucht sind einzige oder kombinierte Suchtformen bei 82,9\% der Frauen in Gruppe V. In dieser Gruppe zeigen $75,6 \%$ der Frauen ein polytoxikomanes Verhalten, in Gruppe I sind es 48,6\%: Intensive Gewalterfahrungen mit sexuellem Missbrauch (Gruppe I) oder frühe seelischemotionale Gewalt (Gruppe V) scheinen zu polytoxikomanem Verhalten zu führen. Den Gebrauch von 4 Suchtmitteln gaben ausschließlich Frauen mit Gewalterfahrung an.

Die gewählten Kombinationen (2 oder mehr Süchte) sind typisch, in der Gruppe I und II sind es neben Alkohol vor allem Essstörungen (Bulimie) oder/und Medikamente, die Gruppe III der älteren Abhängigen kombiniert, wenn überhaupt, Alkohol mit Medikamenten, was auf historisch geprägtes Verhalten hinweist.

Besonders bei den Essstörungen, aber auch beim Medikamentenbeigebrauch muss von einer Unterschätzung ausgegangen werden, da diese zusätzlichen Abhängigkeiten meist nicht bei der Aufnahme, sondern erst im Laufe der Therapie offenbar werden.

Aus psychodynamischer Sicht steht die Bulimie als Problemlösung der Alkoholabhängigkeit näher als den anderen Formen der Essstörungen; bei beiden ist der Anpassungsdruck sehr groß, während bei Mager- und Esssucht, besonders wenn sie mit polytoxikomanem Verhalten verbunden sind, die Protesthaltung mit Selbstverletzungs- oder Selbstzerstörungstendenz deutlicher ist.

\section{Sucht im nahen sozialen Umfeld}

Von 397 Frauen haben 182 (45,8\%) Sucht im häuslichen Umfeld erlebt, bei den 310 Frauen mit Gewalterfahrung traf dies auf $52,9 \%(n=164)$, bei den 87 Frauen ohne Gewalterfahrung auf $20,7 \%(n=18)$ zu. Die höchsten Prozentsätze entfallen auf die Frauen mit Gewalterfahrung in den Gruppen I, II und IV (life event), nämlich 60,2\%, 52,0\% und 75,0\%, Sucht im sozialen Umfeld wird sowohl in der Herkunftsfamilie wie in späteren Partnerschaften geschildert. Bei Gruppe IV müssen die kleinen Fallzahlen berücksichtigt werden, es könnte aber sein, dass die Copingstrategie „Sucht“ für das einschneidende Lebensereignis sozial erlernt wurde. Gruppe III hat, wenn überhaupt, Sucht beim Partner erlebt. In Gruppe V (Essgestörte) wurden ausschließlich die 8 Frauen mit Sucht konfrontiert, die auch Gewalterfahrungen haben. Sucht im nahen sozialen Umfeld scheint demnach eng mit Gewalt verknüpft zu sein.

\section{Psychische Störungen}

Die Frauen wurden bei der Aufnahme nach ambulant oder stationär behandelten oder unbehandelten psychiatrischen Vorerkrankungen befragt, die sie über lange Zeiträume beeinträchtigt haben. $42,1 \%$ aller Frauen $(n=167)$, und zwar $46,1 \%$ $(n=143)$ derjenigen mit und 27,6\% $(n=24)$ derjenigen ohne Gewalterfahrungen, haben schon vor der Entwöhnungsbehandlung an seelischen Störungen gelitten. Am stärksten von allen ist Gruppe I betroffen (67,9\%), die auch die meisten als negativ bewerteten Variablen aufweist. In Gruppe IV (life event) weisen 41,7\%, in Gruppe V (Essgestörte) 41,5\% und in Gruppe II 28,6\% der Frauen seelische Störungen auf. Der geringste Anteil an Frauen mit seelischen Vorerkrankungen findet sich in Gruppe III (9,3\%); diese wird auch erst in höherem Lebensalter abhängig. 
Während Suchtstrategien, neben Suizidversuchen und Depressionen, wie in den Gruppen II, III und IV, als Bewältigungsstrategien auszureichen scheinen, um psychosoziale Defizite und die Erfahrung vorwiegend körperlich-seelischer Gewalt „aufzufangen“, scheinen sich schwere seelische Störungen, besonders Psychosen, posttraumatische Stresssyndrome und Selbstverletzungen bei sexuellem Missbrauch und seelisch-emotinaler Gewalt in der Kindheit, wie in den Gruppen I und V, die auch ein polytoxikomanes Verhalten aufweisen, zu entwickeln. Eine Differenzierung der seelischen Störungen zeigt Tab. 6.

Von den 167 Frauen mit psychischen Störungen wurden seelische Störungen 261-mal benannt, am häufigsten Suizidversuche, nämlich von 62,3\%, Depressionen von $26,3 \%$ und Angststörungen von 22,8\% der Frauen. Bezogen auf alle 397 Frauen sind dies bei den Suizidversuchen 26,2\% der Frauen, alle anderen Störungen kommen bei weniger als $10 \%$ aller Frauen vor.

7,8\% der Frauen mit psychischen Störungen (3,3\% in der Gesamtgruppe) gaben Psychosen (Schizophrenien, einmal Zyklothymie) oder vorbehandelte posttraumatische Stresssyndrome (PTS) an. Gruppe I ist am häufigsten betroffen. Einige dieser Stresssyndrome, insbesondere die multiple Persönlichkeitsstörung, wurden von den Vorbehandelnden als Psychosen fehldiagnostiziert und erwiesen sich als nicht (mit Neuroleptika) behandelbar.

Die psychosomatischen Störungen scheinen mit 9,6\% in der Gesamtgruppe (Anteil an allen psychischen Störungen 15,7\%) deutlich unterschätzt, wie die Entwöhnungsbehandlung im weiteren Verlauf zeigte. Es wird geschätzt, dass mindestens 20-30\% der Frauen unter diesen Störungen leiden.

\section{Diskussion}

Viele der hier als suchtauslösend beschriebenen (und von den Frauen so bewerteten) Variablen sind mit großer Sicherheit auch bei nicht süchtigen Frauen zu finden. Welche Konstellationen letztendlich Sucht bestimmend sind, bleibt unklar, da negative Einflussfaktoren durch positive offenbar gemildert werden können.
Die unterschiedlichen Formen der Gewalt haben sich in dieser Untersuchung als verlässliche Indikatoren für andere assoziierte Lebenserfahrungen und Störungen sowie die Suchtausprägung gezeigt. Die Untersuchung machte aber auch deutlich, dass eine ähnliche Gewalterfahrung bei zwei Frauen von der Frau besser kompensierbar ist, die einen positiven Ausgleich erfahren hat, etwa durch sich gegenseitig unterstützende Geschwister.

Psychische Gewalt und sexueller Missbrauch müssen differenziert werden: Gesellschaftlich akzeptierte Werte wie Ehrgeiz, Ordnung, Leistung oder Aufopferungsfähigkeit werden dann von den untersuchten Frauen als unterdrückend und grenzüberschreitend erlebt, wenn sie als überwiegendes oder ausschließliches Erziehungsziel postuliert werden und alle übrigen Lebensbereiche dominieren. „Sexueller Missbrauch“ kann von den Betroffenen als Zerstörung, aber auch Zuwendung in einer sonst kalten, ablehnenden Welt erlebt werden. Daher erscheint die Möglichkeit zur persönlichen Bewertung durch die Betroffenen besonders wichtig.

Zukünftige Befragungen abhängiger Frauen sollten erst gegen Ende der Therapie stattfinden, wenn früh erlebte Kränkungen erinnert wurden und zusätzliche Süchte offenbar geworden sind.

Der hohe Anteil von Frauen mit Gewalterfahrung ist höchstwahrscheinlich spezifisch für die Klientel frauenspezifischer Einrichtungen, die, bei entsprechend belasteten Frauen, von den Beratungsstellen vorgeschlagen werden [11]. Es wird vermutet, dass sich bei Einbezug von Frauen aus gemischtgeschlechtlich belegten Einrichtungen die prozentuale Verteilung der gebildeten Gruppen anders darstellen würde und die Gruppe der ausschließlich alkoholabhängigen Frauen deutlich größer wäre.

Bei zukünftigen Untersuchungen über die Ursachen der Sucht wäre die Aufhebung der Trennung in legale und illegale Süchte und Essstörungen zu diskutieren, da die Ergebnisse dieser Studie darauf hinweisen, dass es fließende Übergänge zwischen den Suchtarten und -formen gibt und nicht so sehr das Symptom „Sucht“, sondern die Lebenserfahrungen der Frauen die Gruppeneinteilungen plausibel erscheinen lassen.

Tab. 6 Differenzierung psychischer Störungen

\begin{tabular}{|c|c|c|c|c|c|c|c|c|c|c|c|c|c|c|c|c|}
\hline \multirow{2}{*}{$\begin{array}{l}\text { Gruppen } \\
\text { (Anzahl) }\end{array}$} & \multicolumn{2}{|c|}{$\begin{array}{l}\text { Suizid- } \\
\text { versuche }\end{array}$} & \multicolumn{2}{|c|}{$\begin{array}{l}\text { Depres- } \\
\text { sionen }\end{array}$} & \multicolumn{2}{|c|}{ Ängste/Panik } & \multicolumn{2}{|c|}{$\begin{array}{l}\text { psycho- } \\
\text { somatische } \\
\text { Störungen }\end{array}$} & \multicolumn{2}{|c|}{$\begin{array}{l}\text { Selbst- } \\
\text { verletzungen }\end{array}$} & \multicolumn{2}{|c|}{$\begin{array}{l}\text { Belastungs- } \\
\text { störungen }\end{array}$} & \multicolumn{2}{|c|}{$\begin{array}{l}\text { Psychosen/ } \\
\text { posttraum. } \\
\text { Stresssyndr. }\end{array}$} & \multicolumn{2}{|c|}{$\begin{array}{l}\text { Nennungen } \\
\text { gesamt }\end{array}$} \\
\hline & $\mathrm{n}$ & $\%$ & $\mathrm{n}$ & $\%$ & $\mathrm{n}$ & $\%$ & $\mathrm{n}$ & $\%$ & $\mathrm{n}$ & $\%$ & $\mathrm{n}$ & $\%$ & $\mathrm{n}$ & $\%$ & $n$ & $\%$ \\
\hline $\begin{array}{l}\text { Gruppe I } \\
(n=95)\end{array}$ & 63 & 66,3 & 18 & 18,9 & 23 & 24,2 & 22 & 23,2 & 16 & 16,8 & 15 & 15,8 & 11 & 11,6 & 168 & 64,4 \\
\hline $\begin{array}{l}\text { Gruppe II } \\
(n=46)\end{array}$ & 26 & 56,5 & 18 & 39,1 & 11 & 23,8 & 1 & 2,1 & & & & & 2 & 4,3 & 58 & 22,2 \\
\hline $\begin{array}{l}\text { Gruppe III } \\
(n=4)\end{array}$ & 2 & 50,0 & 1 & 25,0 & & & 1 & 25,0 & & & & & & & 4 & 1,5 \\
\hline $\begin{array}{l}\text { Gruppe IV } \\
(n=5)\end{array}$ & 2 & 40,0 & 2 & 40,0 & 1 & 20,0 & 1 & 20,0 & & & & & & & 6 & 2,3 \\
\hline $\begin{array}{l}\text { Gruppe V } \\
(n=17)\end{array}$ & 11 & 64,7 & 5 & 29,4 & 3 & 17,6 & 1 & 5,9 & 4 & 23,5 & 1 & 5,9 & & & 25 & 9,6 \\
\hline alle $=167$ & 104 & 62,3 & 44 & 26,3 & 38 & 22,8 & 26 & 15,7 & 20 & 12,0 & 16 & 9,6 & 13 & 7,8 & 261 & 100 \\
\hline
\end{tabular}


Sucht wird in dieser Untersuchung als Ausdruck oder sichtbares Zeichen einer allgemeineren psychischen Störung im Sinne mangelnder Ich-Stärke gewertet. Sie tritt entweder als einzige Form oder mit anderen psychischen Auffälligkeiten in Erscheinung; beide speisen sich aus denselben Ursachen. Die Frage, ob die eine die Folge der anderen Erkrankung war, erscheint daher unwesentlich. Letztendlich ist zu diskutieren, ob es nicht ratsamer wäre, gemeinsame Einrichtungen für psychisch kranke und suchtkranke Frauen vorzuhalten, als gemischtgeschlechtliche für seelisch Kranke oder Suchtkranke zu favorisieren.

Therapeutisches Handeln muss durch Kenntnisse über Suchtgruppen differenziert werden. Dies betrifft die Dauer der Behandlung, ambulantes oder stationäres Setting, anzuwendende Therapieverfahren, notwendige geschlechtsspezifische Angebote und die sozialtherapeutische Betreuung der Kinder alkoholkranker Frauen/Familien. Auf die spezifischen therapeutischen Bedürfnisse von Frauen wird in den StandardTherapieprogrammen, die auf der Herstellung von Suchtfreiheit bei männlichen Alkoholikern beruhen, bisher nicht ausreichend geachtet, s. a. [12,13].

Primärprävention zur Verhinderung der Entstehung von Abhängigkeit sollte einerseits auf Wahrnehmungsschulung, Kompetenzentwicklung und -stärkung bei Pädagogen, Mitarbeitern der Familienhilfen, anderen sozialen Professionen sowie Kinder-, Hausärzten und Gynäkologen zur frühen Identifikation suchtbahnender Faktoren gerichtet sein, spezifische Beratungs- und Betreuungsangebote für gefährdete Kinder/Jugendliche müssen andererseits, speziell in Schulen, stärker etabliert werden.

\section{Literatur}

${ }^{1}$ Preuß UW, Schröter A, Soyka M. Typologien der Alkoholkrankheit - ein kritischer Vergleich. Sucht, 1997; 43: 92-103 (2)

${ }^{2}$ Schuckit MA, Daeppen JB, Tipp JE, Hesselbrock M, Bucholz KK. The clinical course of alcohol-related problems in alcohol dependent and nonalcohol dependent drinking women and men. J Stud Alcohol 1998; 59: 581-590 (5)

${ }^{3}$ Miller BA, Downs WR, Testa M. Interrelationships between victimization experiences and women's alcohol use. J Stud Alcohol Suppl 1993; 11: 109-117 (9)

${ }^{4}$ Swett C, Halpert M. High rates of alcohol problems and history of physical and sexual abuse among women inpatients. Am J Drug Alcohol Abuse 1994; 20: 263-272 (2)

${ }^{5}$ Spak L, Spak F, Allebeck P. Factors in childhood and youth predicting alcohol dependence and abuse in Swedish women: findings from a general population study. Alcohol Alcohol 1997; 32: 267-274 (3)

${ }^{6}$ Wilsnack SC, Vogeltanz ND, Klassen AD, Harris TR. Childhood sexual abuse and women's substance abuse: national survey findings. J Stud Alcohol 1997; 58: 264-271 (3)

${ }^{7}$ Fleming J, Mullen PE, Sibthorpe B, Attewell R, Bammer G. The relationship between childhood sexual abuse and alcohol abuse in women - a case-control study. Addiction 1998; 93: 1787-1798 (12)

${ }^{8}$ Küfner H, Duwe A, Schumann J, Bühringer G. Prädiktion des Drogenkonsums und der Suchtentwicklung durch Faktoren in der Kindheit: Grundlagen und Ergebnisse einer empirischen Studie. Sucht 2000; 46: 32-53 (1)

${ }^{9}$ Langeland W, Hartgers C. Child sexual and physical abuse and alcoholism: a review. J Stud Alcohol 1998; 59: 336-348 (3)
${ }^{10}$ Vogt I. Alkoholikerinnen. Freiburg: Lambertus, 1994

${ }^{11}$ Zenker C. Frauenspezifische Therapieeinrichtungen. In: Gölz J (Hrsg). Moderne Suchtmedizin. Stuttgart, New York: Thieme, 1998

12 Goldberg ME. Substance-abuse in women: false stereotyppes and real needs. Soc Work, 1995; 40: 789-798 (6)

${ }^{13}$ Spak L, Spak F, Allebeck P. Sexual abuse and alcoholism in a female population. Addiction 1998; 93: 1365-1373 (9)

Prof. Dr. med. Christel Zenker

Ilsenburger Straße 6

28205 Bremen

E-mail: chzenker@fh-muenster.de 